\title{
BMJ Open Standardised patient encounters to improve quality of counselling for pre- exposure prophylaxis (PrEP) in adolescent girls and young women (AGYW) in Kenya: study protocol of a cluster randomised controlled trial
}

\author{
Anna Larsen (1) , ${ }^{1,2}$ Kate S Wilson, ${ }^{1}$ John Kinuthia, ${ }^{1,3}$ G John-Stewart, ${ }^{2,4,5}$ \\ BA Richardson, ${ }^{6}$ Jillian Pintye, ${ }^{1}$ Felix Abuna, ${ }^{3}$ Harison Lagat, ${ }^{3}$ Tamara Owens, ${ }^{7}$ \\ Pamela Kohler 8
}

To cite: Larsen A, Wilson KS, Kinuthia J, et al. Standardised patient encounters to improve quality of counselling for preexposure prophylaxis (PrEP) in adolescent girls and young women (AGYW) in Kenya: study protocol of a cluster randomised controlled trial. BMJ Open 2020;10:e035689. doi:10.1136/ bmjopen-2019-035689

- Prepublication history and additional material for this paper are available online. To view these files, please visit the journal online (http://dx.doi org/10.1136/bmjopen-2019035689).

Received 12 November 2019 Revised 03 February 2020 Accepted 26 May 2020

A) Check for updates

(c) Author(s) (or their employer(s)) 2020. Re-use permitted under CC BY-NC. No commercial re-use. See rights and permissions. Published by BMJ.

For numbered affiliations see end of article.

Correspondence to Anna Larsen; annalar@uw.edu

\section{ABSTRACT}

Introduction Adolescent girls and young women (AGYW) in sub-Saharan Africa are at high risk of HIV acquisition. Pre-exposure prophylaxis (PrEP) demonstration projects observe that AGYW uptake and adherence to PrEP during risk periods is suboptimal. Judgemental interactions with healthcare workers (HCW) and inadequate counselling can be barriers to PrEP use among AGYW. Improving HCW competency and communication to support PrEP delivery to AGYW requires new strategies.

Methods and analysis PrEP Implementation for Young Women and Adolescents Program-standardised patient (PrIYA-SP) is a cluster randomised trial of a standardised patient actor (SP) training intervention designed to improve HCW adherence to PrEP guidelines and communication skills. We purposively selected 24 clinics offering PrEP services under fully programmatic conditions in Kisumu County, Kenya. At baseline, unannounced SP 'mystery shoppers' present to clinics portraying AGYW in common PrEP scenarios for a cross-sectional assessment of PrEP delivery. Twelve facilities will be randomised to receive a 2-day training intervention, consisting of lectures, roleplaying with SPs and group debriefing. Unannounced SPs will repeat the assessment in all 24 sites following the intervention. The primary outcome is quality of PrEP counselling, including adherence to national guidelines and communication skills, scored on a checklist by SPs blinded to intervention assignment. An intention-to-treat (ITT) analysis will evaluate whether the intervention resulted in higher scores within intervention compared with control facilities, adjusted for baseline SP scores and accounting for clustering by facility. We hypothesise that the intervention will improve quality of PrEP counselling compared with standard of care. Results from this study will inform guidelines for PrEP delivery to AGYW in lowresource settings and offer a potentially scalable strategy to improve service delivery for this high-risk group. Ethics and dissemination The protocol was approved by institutional review boards at Kenyatta National Hospital and University of Washington. An external
Strengths and limitations of this study

- The cluster randomised controlled trial design offers rigorous assessment of a clinical training intervention using standardised patient actors (SPS) to improve pre-exposure prophylaxis (PrEP) for HIV delivery to adolescent girls and young women in Western Kenya.

- The study uses SPs, a validated method for evaluating health provider performance, to assess communication skills and compliance with Kenyan national PrEP guidelines.

- The use of SPs to support provider training is a novel approach to improving clinical counselling in lowresource settings.

- The pragmatic trial design offers the potential for application within the health system.

- Study activities are subject to delays from public holidays, provider strikes, PrEP stock-outs and staff turnover; thus intervention fidelity may be limited by real-world circumstances.

advisory committee monitors social harms. Results will be disseminated through peer-reviewed journals and presentations.

Trial registration number NCT03875950

\section{INTRODUCTION}

Adolescent girls and young women (AGYW) aged 15-24 years old in high HIV-burden settings in sub-Saharan Africa (SSA) have an eightfold higher risk of HIV acquisition than men of the same age group, ${ }^{1}$ and in 2019 an estimated 320000 new HIV infections occurred in AGYW globally. ${ }^{2}$ Pre-exposure prophylaxis (PrEP) has been shown to be highly efficacious to prevent HIV transmission 
among adults, when adherence is high, ${ }^{3-8}$ and it is a promising HIV prevention tool for groups at high risk of HIV acquisition including AGYW.

Kenya is among the first countries in Africa to offer guidelines to support delivery of PrEP to AGYW ${ }^{9}$ and conduct large PrEP delivery demonstration projects in public sector maternal and child health $(\mathrm{MCH})$ and family planning (FP) clinics. PrEP initiation and continuation among AGYW is higher in real-world setting demonstration projects than randomised controlled trials (RCTs) among African women, yet important barriers remain preventing optimal PrEP use among this group. ${ }^{10}$ One of the major health system-level barriers to adolescent engagement in sexual and reproductive health services, including PrEP, is poor interactions with healthcare workers (HCW), specifically fear of judgement and lack of confidentiality. ${ }^{12-18}$ Similarly, HCWs report lacking knowledge and skills in working effectively with this population, especially concerning HIV prevention. ${ }^{12-21}$ Improving the quality of PrEP counselling tailored to AGYW is critical to support optimal use of PrEP.

Standardised patient actors (SPs) are an evidence-based training method for improving provider competency and quality of care. ${ }^{22-26}$ SPs have been used to train providers in a variety of clinical skills ${ }^{27}$ leading to improved patient outcomes. ${ }^{28}{ }^{29}$ Increasingly, SPs are used as 'mystery shoppers' posing as real patients to assess provider compliance with clinical guidelines. SPs have assessed provider competencies in tuberculosis care, ${ }^{30}$ sexual health assessment ${ }^{23}$ and HIV care, ${ }^{24}$ with emerging evidence from lowincome and middle-income countries including South Africa and Kenya. SPs are especially effective at improving and assessing HCW skills in patient-centred communication, a key component of quality of care,${ }^{31}$ and adherence to clinical guidelines. ${ }^{24}$

In a context of rapid roll-out of PrEP services to AGYW, it is important to evaluate practical strategies to ensure quality service delivery. A clinical training intervention using SPs may improve quality of PrEP delivery for AGYW, and ultimately scale-up and uptake of this effective prevention tool. This cluster RCT (cRCT) will evaluate impact of a SP training intervention on the quality of PrEP counselling at the visit level, including adherence to national guidelines ${ }^{32}$ and communication skills, provided by HCWs delivering PrEP to AGYW. Results from this study could demonstrate an evidence-based, scalable intervention to improve delivery of PrEP as an attractive HIV prevention option for AGYW in Africa, with the ultimate goal of reducing HIV acquisition among this priority population.

\section{METHODS AND ANALYSIS \\ Study design}

The Simulated Patients to Improve PrEP Counselling for AGYW in Kenya, 'PrEP Implementation for Young Women and Adolescents Program-SP' (PrIYA-SP) study aims to evaluate the effectiveness of a clinical training programme using SPs to improve PrEP counselling to AGYW. This cRCT compares HCW adherence to national PrEP delivery guidelines and communication skills between the intervention and comparison facilities assessed post SP-led training intervention. PrEP delivery by HCW is evaluated via standardised checklists prepared by SPs acting as 'mystery shoppers' during unannounced PrEPseeking encounters. PrEP delivery scores are measured at the SP encounter level and compared between intervention and control sites, clustering by facility. Comparison facilities do not receive the clinical training.

The present study extends the work of the PrIYA. This was a 2-year implementation project to reach AGYW at high risk for HIV acquisition through integrated delivery of PrEP within routine MCH and FP systems. ${ }^{19} 33$ PrIYA was implemented from June 2017 to December 2018 in 16 facilities (11 public, 4 faith-based and 1 private) in Kisumu County, Kenya. PrIYA was followed by a PrEP mentorship programme in 21 additional sites involving in-clinic guidance from former PrIYA nurses to HCW in non-PrIYA sites about best practices for delivering PrEP to AGYW. Following conclusion of the PrEP mentorship programme, we initiated the present follow-on study in 24 former PrIYA and PrEP mentorship sites.

\section{Study sites and population}

\section{Eligibility criteria for facilities and HCW}

The study is conducted at 24 large public health facilities, including former PrIYA Program and former PrIYA mentorship sites, that provide PrEP care and counselling services to AGYW in Kisumu County, Kenya (figure 1). Thirty-seven facilities were evaluated for inclusion, of which 24 were purposively selected based on expected patient volume of at least two AGYW seeking PrEP per week. All facility managers approached for inclusion agreed to participate in the study. Facilities with ongoing PrEP interventions involving PrEP delivery by research

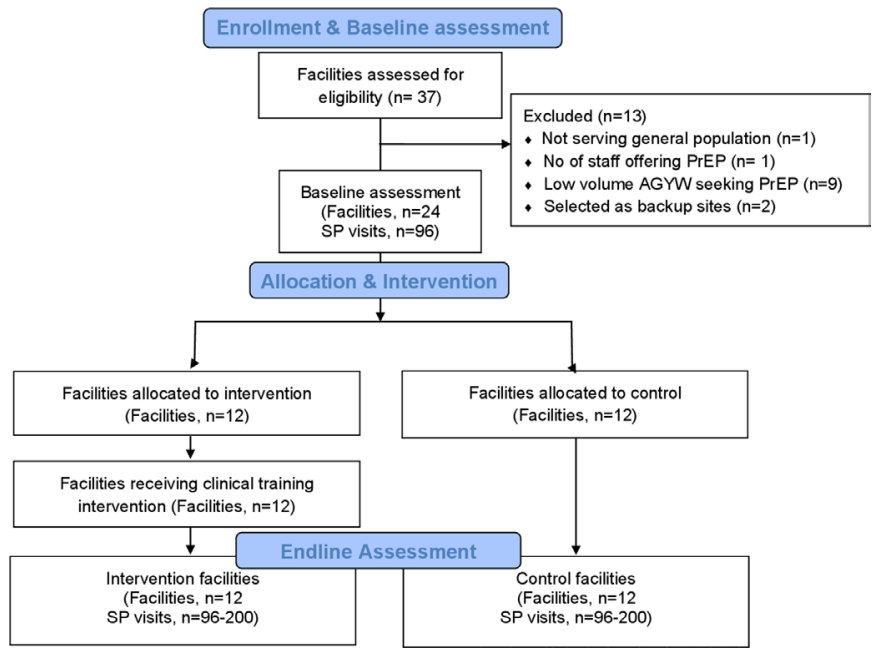

Figure 1 Adapted CONSORT diagram for PrIYA-SP. AGYW, adolescent girls and young women; PrEP, pre-exposure prophylaxis; PrIYA, PrEP Implementation for Young Women and Adolescents Program; SP, standarised patient. 
personnel were excluded. Sampling aimed for an even distribution across facility levels (county, subcounty and health centre), settings (urban, peri-urban and rural) and types (public and private/faith-based) to ensure generalisability of results.

All HCWs, who provide PrEP delivery services to AGYW, are current employees at the study sites, are 18 years or older and are able to provide informed consent, are approached for enrolment. Within Kenyan FP and MCH settings, nurses, clinical officers and doctors predominantly comprise the HCW cadres trained to deliver PrEP, with other cadres such as HIV Testing Services counsellors less frequently involved. Any HCWs who are working as research staff on another AGYW trial or intervention are excluded. We anticipated that $10 \mathrm{HCWs}$ or fewer per site will be eligible, totalling up to 240 HCWs.

\section{Patient and public involvement}

This study was designed without public involvement. Members of the public were not invited to comment on the study design, were not consulted to develop patientrelevant outcomes and were not involved in designing a dissemination plan. Results of this study will be disseminated through peer-reviewed journals, presentations at local and international conferences to national and global policymakers, community members and participants.

\section{Randomisation}

Facility cluster randomisation is conducted using a stratified approach based on facility level (county/subcounty hospital vs health centre) and facility patient volume of PrEP clients ( $\geq 5$ female PrEP clients per week (high volume) vs $<5$ female PrEP clients per week (low volume)) to ensure balance by arm. Stratification groups were selected to further reduce potential imbalance between intervention and control facilities. We do not expect that facility level and volume of AGYW seeking PrEP would be meaningfully correlated because facility-level patient volume of PrEP clients is not determined by facility level within the health system in Western Kenya. Each facility is categorised by facility level and patient volume of PrEP clients prior to randomisation and assigned to four groups. The randomisation assignment is generated by a biostatistician, resulting in 12 facilities allocated to

Table 1 Facility characteristics for PrIYA-SP restricted randomisation

\begin{tabular}{lll}
\hline Facility level & Patient volume* $^{*}$ & Facilities (n) \\
\hline Hospital & High volume & 8 \\
Hospital & Low volume & 3 \\
Health centre & High volume & 6 \\
Health centre & Low volume & 7 \\
\hline
\end{tabular}

*High volume of female PrEP clients per week: $\geq 5=$ high volume and $<5=$ low volume.

PrEP, pre-exposure prophylaxis; PrIYA, PrEP Implementation for Young Women and Adolescents Program; SP, standarised patient. the clinical training intervention arm and 12 facilities allocated to standard of care (table 1). We do not expect contamination between intervention and control sites, as selected facilities are geographically located with sufficient distance from each other to limit HCW interaction between facilities. Furthermore, the intervention takes place over a short time period (6 months) to minimise staff turnover.

\section{Blinding}

Given the study design as a cRCT of facilities with staff who will be aware of receiving training or not, it is impossible to blind all study team members and participants to randomisation assignments. However, we are implementing procedures to minimise the number of individuals who are unblinded to study assignment and outcomes. Unannounced SPs are blinded to facility allocation. Data monitoring does not include information about study end points disaggregated by facility or study arm. Only the biostatistician, data manager and designated field staff have access to data on study outcomes by study arm or facility. These reports are viewed exclusively during closed External Advisory Committee sessions, which do not include study team members. One month before the trial, the biostatistician will provide a list of the intervention facilities to study team members leading field implementation. In their roles as 'mystery shoppers', unannounced SPs are trained to be indistinguishable by HCW from real AGYW seeking services within study sites, thus HCW are in essence 'blinded' to SPs. During the classroom role-play encounters within the clinical training intervention, SPs are known to the HCW as actresses. To reduce discovery of unannounced SPs in the postintervention evaluation, different actresses from those participating in the baseline assessment and training intervention are employed as 'mystery shoppers'.

\section{Study procedures}

\section{Standardised patient actor selection and training}

Professional Kenyan actors are hired through an agreement with a Kenyan casting agency and selected to be representative of AGYW in Western Kenya. Actors are trained in the SP methodology during a 5-day training facilitated by a simulation expert. Training includes didactic demonstrations, role-play, paired practice and small group discussions focused on preparing SPs to portray the patient cases realistically and accurately. Practice interactions between SPs and HCWs are video-taped and discussed. Consultation during the training informs refinement of case portrayal, use of measurement tools, as well as revisions to the case scripts to ensure accuracy, feasibility and believability in field implementation. Two SPs are assigned to each case script for a total of eight SPs who consistently perform cases at all health facilities throughout the baseline assessment and clinical training intervention. After the intervention period, a new group of SPs are hired and trained to perform unannounced 
case scripts in the same manner for the postintervention evaluation to reduce chance of SP discovery by HCWs.

\section{Case script development}

Standardised case scripts for both unannounced assessments and training encounters are developed by an SP expert, Kenyan and US clinicians and adolescent and young adult researchers to represent common experiences and challenges faced by AGYW seeking PrEP in Western Kenya and our prior studies. ${ }^{23}{ }^{24}$ Each case follows a standard format, including a case summary, background highlighting chief complaint, medical and social history and actor prompts. Development of the cases was informed by multiple sources through an iterative process. Themes and insights were incorporated from qualitative themes emerging from previously conducted in-depth interviews, focus group discussions among AGYW, feedback from PrIYA nurses with 2 years of experience delivering PrEP to AGYW in Kisumu, focus group discussions among a community advisory board, perspectives from PrEPexperienced study staff, key informant experiences and national guidelines. ${ }^{32} 33$ Four unannounced and six classroom training case scripts were developed, representing unique PrEP use circumstances, including but not limited to sexual activity among young adolescents, transactional sex, multiple concurrent partners and having an HIVpositive partner.

\section{Recruitment and enrolment}

The study team obtained permission from the National AIDS \& STI Control Programme and local county and district officials to enrol selected study facilities in the PrIYA-SP study. Leadership personnel at each of the study facilities are offered information about the study and invited to provide written informed consent (online supplementary file 1) to participate in a health facility survey. We consult facility managers to identify HCWs delivering PrEP to AGYW and to negotiate release from work to participate in survey data collection, and if randomised, the training intervention. Identified HCWs are invited by a study interviewer to learn more about the study and, if interested, provide written informed consent (online supplementary file 1). We track HCWs over time to monitor staff turnover and provide ongoing opportunities for informed consent by incoming HCWs delivering PrEP to AGYW.

Baseline data collection through surveys and unannounced patient actor encounters

At enrolment, surveys are administered to consenting facility managers by study staff to obtain baseline sitelevel characteristics describing staffing, presence of national and AGYW-specific guidelines and PrEP service availability (table 2). Study staff also administer surveys to HCWs to ascertain demographics, training history, beliefs about HIV, ${ }^{34}$ AGYW and $\mathrm{PrEP}^{20}$; self-reported competency in PrEP delivery to AGYW and self-reported knowledge of PrEP services. Unannounced SP actors, or 'mystery shoppers', present to the 24 study sites at baseline posing as AGYW seeking PrEP. Each facility receives four SP encounters at baseline (one visit per case script). During the clinic encounter, SPs perform their assigned scripted scenario with enrolled HCWs, as if they were a real patient. After the encounter, the SP fills out a post-consultation checklist to assess HCW adherence to national guidelines and communication skills using standardised questions with binary ('done'/'not done') or four-point Likert scale response options. The checklist includes a section eliciting open-ended responses from SPs about each encounter. SPs are trained in how to fill out the checklist using a rubric and checklists are reviewed by a study team member after each encounter to ensure completeness and consistency across SPs. Study staff coordinate with the health facility to remove any false 'patient' information provided during the SP encounter from clinic forms and registers.

\section{Intervention}

Enrolled HCWs from the 12 study sites randomised to receive the clinical training intervention are invited to attend 2-day training events among groups of 5-10 HCWs, totalling 20 repeated training events. All HCWs, regardless of cadre, will receive the same 2-day training to ensure consistency of exposure to PrEP delivery guidelines and patient-provider communication content. During the trial period, HCW maintain normal daily functions aside from participation in the 2-day training event. These events involve a combination of didactic lectures providing background information about the PrIYA-SP trial, adolescent health and development, Kenyan national guidelines for HIV prevention and PrEP delivery among AGYW and structuring a high-quality patient-provider encounter. Interactive group activities include a values clarification exercise and patient-centred communication activity which focus on understanding the patient's perspective, verbal and nonverbal communication, expressing empathy and shared decision-making. ${ }^{31}$ These activities allow HCW to practise reducing the influence of their personal beliefs within patient care and engaging patients in their own health decision-making processes.

Training materials for the intervention are informed by widely used frameworks for clinical communication skills and high-quality patient-provider interactions, ${ }^{22} 31$ 35-42 the current Kenyan national guidelines for PrEP delivery ${ }^{43}$ and qualitative interviews with AGYWs regarding PrEP seeking experiences conducted by study team members. The clinical training intervention is adapted from the training intervention implemented in the SPEED trial-a stepped-wedge RCT evaluating the effect of a SP-led training intervention on adolescent retention in HIV care in Kenya (details published previously). ${ }^{24}$

HCW role-play with SPs to enact six case scripts depicting common experiences of AGYW seeking PrEP counselling. Each encounter is video-recorded and timed. During the informed consent process, HCW agree to video-recording 
Table 2 Adapted spirit diagram for PrIYA-SP trial

\begin{tabular}{|c|c|c|c|c|c|c|}
\hline \multirow[b]{2}{*}{ Time point } & \multirow{2}{*}{$\begin{array}{l}\text { Enrolment } \\
\text { Month } \\
0\end{array}$} & \multirow{2}{*}{$\begin{array}{l}\text { Baseline } \\
\text { Months } \\
0-6\end{array}$} & \multicolumn{4}{|c|}{ Trial implementation } \\
\hline & & & Months 6-9 & Months 9-12 & Months 12-15 & Months 15-18 \\
\hline Eligibility screen & $x$ & & & & & \\
\hline Informed consent & $x$ & & & & & \\
\hline $\begin{array}{l}\text { Clinical training } \\
\text { intervention (intervention } \\
\text { arm only) }\end{array}$ & & & $x$ & $X$ & & \\
\hline \multicolumn{7}{|l|}{ Assessments } \\
\hline $\begin{array}{l}\text { Facility manager survey } \\
\text { Respondent } \\
\text { characteristics } \\
\text { HIV prevention training } \\
\text { HIV prevention/care } \\
\text { guidelines } \\
\text { PrEP for adolescents } \\
\text { and young adults } \\
\text { PrEP registry data } \\
\text { collection }\end{array}$ & & $x$ & & & & \\
\hline $\begin{array}{l}\text { Healthcare worker } \\
\text { survey } \\
\text { Demographics and } \\
\text { training history } \\
\text { Beliefs about AGYW } \\
\text { and PrEP } \\
\text { Beliefs about HIV } \\
\text { Self-rated competency } \\
\text { in PrEP delivery to } \\
\text { AGYW } \\
\text { Knowledge of PrEP } \\
\text { services }\end{array}$ & & $x$ & & & $x$ & $x$ \\
\hline $\begin{array}{l}\text { SP training encounter } \\
\text { checklist } \\
\text { PrEP guidelines } \\
\text { adherence } \\
\text { Communication skills } \\
\text { Interpersonal skills }\end{array}$ & & & $x$ & $x$ & & \\
\hline
\end{tabular}

AGYW, adolescent girls and young women; PrEP, pre-exposure prophylaxis; PrlYA, PrEP Implementation for Young Women and Adolescents Program; SP, standardised patient actor.

during the training sessions, and video equipment is readily visible in role-play spaces such that HCW are aware of the recording. HCW may opt to sign a video and photo release form premising use of the videos in educational or dissemination settings beyond the training event. Case scripts from the baseline unannounced visits are adapted for appropriate application in the training intervention. Following each role-play encounter, SPs complete a training checklist evaluating HCW adherence to PrEP guidelines, communication skills and interpersonal skills. Training concludes with review of filmed SP role-play sessions with facilitated group debriefing to synthesise feedback and takeaway messages.

Overall, the clinical training intervention is developed to accommodate a mixed skill set within diverse HCW 


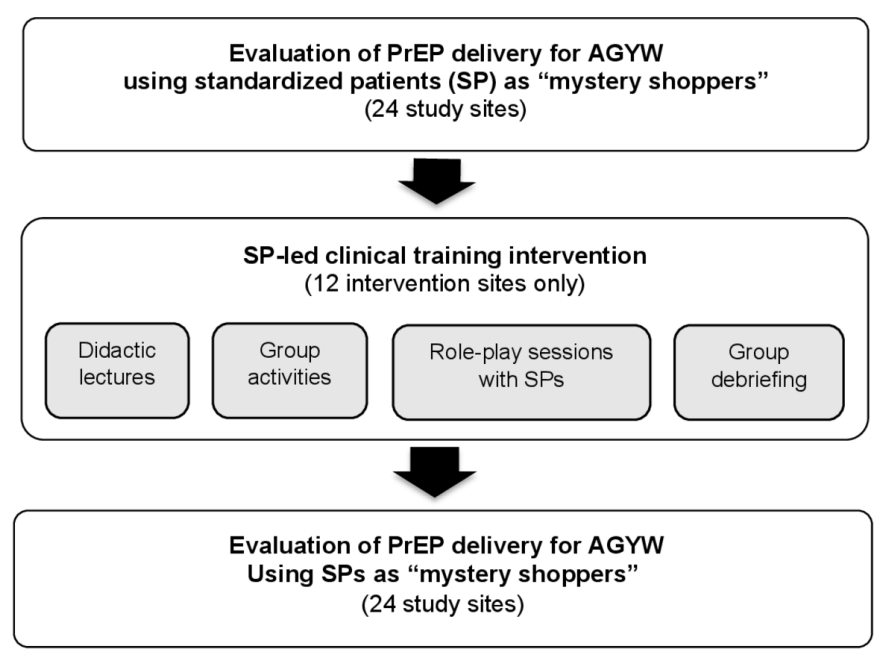

Figure 2 PrIYA-SP trial schematic. AGYW, adolescent girls and young women; PrEP, pre-exposure prophylaxis; PrIYA, PrEP Implementation for Young Women and Adolescents Program; SP, standarised patient.

cadres that could be applied and sustained across the health system by the Kenyan Ministry of Health.

\section{Monitoring intervention fidelity}

We monitor intervention fidelity throughout the trial by evaluating SP actor fidelity to assigned PrEP case scripts. Study staff review a random sample of $10 \%$ of video-taped encounters at regular intervals throughout training intervention implementation, measuring actor fidelity by completing a standardised checklist adapted for this study. Fidelity checklists and scores inform refresher training for SPs throughout implementation of the training intervention. Individual training for actors requiring further support are facilitated as needed. Standard operating procedures and standardised training materials are consistently used for each training session. We track HCW completion of the training intervention and retention in study facilities over time.

\section{Data collection for intervention evaluation}

Once the training intervention is complete for all sites in the intervention arm, study staff re-administer the HCW surveys at all 24 facilities (figure 2). The new group of SPs are hired and trained to perform case scripts, and subsequently SPs repeat the unannounced assessments conducted during the baseline evaluation at all 24 intervention and control sites. Case scripts are updated from those used at baseline and in the training intervention to minimise risk of SP discovery by HCWs. Postintervention checklists and scores used to measure quality of PrEP counselling at each SP encounter are the same as in the baseline evaluation.

\section{Data management}

Data are collected by trained study staff into REDCap-an encrypted, web-based data management application-using password-protected electronic tablets to protect participant confidentiality. The Kenya-based data manager oversees data entry, management and monitoring throughout the study and a Seattle-based statistical team oversees data cleaning, reporting and interim analyses. All data, including video-recordings, are stored on a secure server at the University of Washington throughout the trial and for at least 3 years after trial completion. A Data Monitoring Committee is not needed as this trial is low risk to study participants. An External Advisory Committee reviews trial progress and social harms.

\section{Outcome measures}

The primary outcome of interest for this study is quality of PrEP counselling provided by HCWs delivering PrEP to AGYW for HIV prevention in Kenya, measured at each unique SP encounter and compared between intervention and control sites (table 3). We define quality as the total score from the SP unannounced checklist, which includes subscores for domains of adherence to national PrEP guidelines and communication skills. The checklist contains 12 questions assessing adherence to PrEP guidelines using binary 'done/not done' response options to indicate whether the PrEP counselling message was delivered by the provider during the SP unannounced visit (subscore range: 0-12) according to the Kenyan National AIDS \& STI Control Programme guidelines. ${ }^{43}$ Seven questions assess communication quality with four scaled response options (strongly agree, agree, disagree and strongly disagree) (subscore range: 0-21). Since there are few standard tools to assess patient-provider communication about HIV prevention with young people, questions to assess communication quality were informed by guidelines and tools used in other populations ${ }^{22}{ }^{35-42}$ and adapted for this population. Higher total scores represent higher quality of PrEP counselling (overall score range: 0-33) and are rescaled to a percentage. Quality of PrEP counselling was selected as the primary outcome for this study as it represents an important proximate outcome of the training intervention to improve PrEP services for AGYW which results from an intervention to improve HCW counselling. This may in turn improve AGYW adherence. PrEP uptake and adherence among AGYW, and these outcomes will be evaluated as other important outcomes of programmatic PrEP delivery in other related work.

The secondary outcome for this study is quality of PrEP counselling by HCWs during the classroom-simulated encounters in interpersonal skills, communication skills and adherence to the national guidelines for PrEP delivery comparing mean score percentage from the first and last SP encounters. The interpersonal skills measure includes four dimensions (interviewing and collecting information, counselling and delivering information, rapport and personal manner). Empathy is assessed indirectly as a construct within the interpersonal skills assessment, measured as a combination of skills including active listening and validation. The checklist assesses interpersonal skills with 4 scaled response options (1-4 with higher value indicating higher quality interpersonal 
Table 3 Primary and secondary outcomes and data sources for PrIYA-SP trial

\begin{tabular}{|c|c|c|c|c|}
\hline Outcome measure & Data source* $^{*}$ & Facilities assessed & Comparison & Timing of assessment \\
\hline $\begin{array}{l}\text { Primary outcome: } \\
\text { Quality of PrEP counselling } \\
\text { mean score percentage } \\
\text { from unannounced SP visits }\end{array}$ & $\begin{array}{l}\text { SP unannounced visit } \\
\text { checklists from endline } \\
\text { assessment }^{\star}\end{array}$ & $\begin{array}{l}\text { All intervention and } \\
\text { control sites }\end{array}$ & $\begin{array}{l}\text { Intervention vs control } \\
\text { sites }\end{array}$ & $\begin{array}{l}\text { Postintervention } \\
\text { evaluation }\end{array}$ \\
\hline $\begin{array}{l}\text { Secondary outcome: } \\
\text { Change in interpersonal } \\
\text { skills mean score } \\
\text { percentage among } \\
\text { intervention participants } \\
\text { between the first and last } \\
\text { session }\end{array}$ & SP training checklist* & $\begin{array}{l}\text { Intervention arm sites } \\
\text { only }\end{array}$ & $\begin{array}{l}\text { Initial training SP } \\
\text { encounter to final } \\
\text { training SP encounter }\end{array}$ & Intervention \\
\hline $\begin{array}{l}\text { Other outcome: } \\
\text { Self-rated HCW PrEP } \\
\text { competency, attitudes } \\
\text { towards AGYW }\end{array}$ & $\begin{array}{l}\text { Healthcare worker } \\
\text { survey }\end{array}$ & $\begin{array}{l}\text { All intervention and } \\
\text { control sites }\end{array}$ & $\begin{array}{l}\text { Intervention vs control } \\
\text { sites }\end{array}$ & $\begin{array}{l}\text { Postintervention } \\
\text { evaluation }\end{array}$ \\
\hline
\end{tabular}

*SP unannounced visit checklist and SP training checklist are available in online supplementary file 1.

AGYW, adolescent girls and young women; HCW, healthcare workers; PrEP, pre-exposure prophylaxis; PrIYA, PrEP Implementation for Young Women and Adolescents Program; SP, standardised patient.

skills) developed from the Van Zanten interpersonal skills assessment tool $^{42}$ (subscore range: 4-16). We compute mean score percentage for training intervention scores stratified by case script and from first to last case as a secondary outcome.

\section{Sample size calculation}

Given the study is conducted in 24 facilities comprising the total number of clusters, we used an a priori established expected baseline quality of PrEP counselling to estimate sample sizes to detect a $10 \%$ point difference between the intervention and control arm with $80 \%$ power assuming a type 1 error rate of 0.05 and a two-sided test. Under these assumptions, if quality of PrEP counselling is $60 \%$ in the control arm, with SD of $17.7 \%$ and assumed coefficient of variation of 0.15 between SP encounters, we would need an estimated 120 total SP encounters overall (6 SP encounters per site).

\section{Statistical methods and analysis}

The primary analyses will use intention to treat (ITT) to evaluate whether the clinical training intervention using SPs results in higher quality of PrEP counselling scores at SP encounters taking place in intervention facilities compared with control facilities. The ITT analysis assumes that HCWs in facilities randomised to receive the clinical training intervention are 'exposed' to the training, and that HCWs in facilities randomised not to receive the training intervention are 'unexposed' to the training. We use a CONSORT diagram (figure 1) to indicate the number of facilities and HCWs enrolled by study arm during the trial, numbers excluded and reasons for exclusion. Descriptive statistics describing baseline characteristics of facilities by study arm will be presented to assess whether balance of these factors was achieved through randomisation.
Generalised linear mixed models (GLMMs) will be used to compare postintervention quality of PrEP counselling score percentage from SP encounters between the intervention facilities and comparison facilities. (ie, control arm). We will estimate the effect of the training intervention on the individual SP encounter level, using a GLMM with a Gaussian distribution and identity link, accounting for facility cluster and SP as random effects. These models will be adjusted for baseline quality of PrEP counselling score items that differ between study arms ( $p$ value $<0.05$ ) ascertained using checklists completed by SP actors based on their assessment of care received by HCWs during unannounced SP encounters. This analytical approach allows individual-level outcomes per SP encounter to be modelled while accounting for correlation by facility-level cluster and SP. Regression coefficients and 95\% CIs will be estimated with a two-sided alpha of $5 \%$. In sensitivity analyses, we will evaluate the intervention effect on individual components of PrEP guideline adherence and communication quality in separate GLMMs as well as differences in overall mean percentage scores between cases, where case is included as a fixed effect.

\section{Ethics and dissemination}

The PrIYA-SP study is registered at clinicaltrials.gov. This study was approved by the University of Washington Institutional Review Board and Kenyatta National Hospital Ethics and Research Committee. Changes to the protocol are reviewed by both institutions prior to implementation and appropriate updates are made to clinicaltrials. gov. Any changes to the protocol are communicated to coinvestigators and study staff through change memos. Results from this study will be disseminated through peerreviewed journals, presentations at local and international 
conferences to national and global policymakers, community members and participants.

\section{DISCUSSION}

There is high global commitment to reducing HIV incidence among AGYW and a mounting evidence base that more research on PrEP delivery is needed to maximise the real-world effectiveness of this powerful HIV prevention tool. However, there are few interventions to improve the current quality of PrEP services for AGYW in high HIV-burden settings which may influence uptake and adherence. It is therefore important to evaluate novel and potentially scalable strategies to improve adherence to national PrEP guidelines and communication skills among HCW delivering PrEP to this population. The PrIYA-SP study fills a gap in evidence of HCW training programmes in resource-limited settings to improve HIV prevention services for AGYW. ${ }^{44} 45$ Our use of SPs to assess quality of PrEP delivery and as part of a clinical training intervention is novel. We use a randomised trial to evaluate the outcome of quality of PrEP services while adapting to schedules and services of the facilities. This study is responsive to the global motivation to reduce HIV acquisition among $\mathrm{AGYW}^{46}$ by reducing barriers to PrEP uptake, as well as the Kenyan Ministry of Health request for novel interventions to inform the implementation of new adolescent-friendly HIV service guidelines. ${ }^{47}$ If the SP-led clinical training intervention is effective at improving quality of PrEP counselling for AGYW, SP-led training interventions could be adopted as a recommended approach by Ministries of Health and PrEP scale-up initiatives within similar settings to expand improvements in quality PrEP delivery for AGYW.

\section{Limitations}

This study has limitations. As a pragmatic trial taking place within the Kenyan health system, study activities are subject to delays due to public holidays, health provider strikes, PrEP stock-outs and other reasons for staff shortages within facilities. Staff turnover is likely within this context, which could result in turnover of PrIYASP-trained HCWs and potentially diluted intervention effectiveness. We monitor staff shortages and turnover through operational tracking processes and will account for these changes in the analysis. Finally, our primary outcome depends on the quality of SP recall after the unannounced visits when completing the checklist with study staff. To minimise the potential for error in recall, the consultant-led SP training includes tactics for remembering details of interactions and study staff administer checklists to SPs immediately following unannounced visits.

\section{CONCLUSION}

This training intervention for HCWs involving SPs has the potential to promote high-quality, patient-centred HIV prevention services for AGYW by providing HCWs with improved competency in Kenyan guidelines for PrEP delivery and enhanced communication skills. This study fills a need cited by HCW for expanded skill sets ${ }^{14}$ and a desire cited by AGYW for respectful, ${ }^{16} 1848$ and informative care. ${ }^{17}$ This increase in quality of care delivery may, in turn, improve PrEP uptake and continuation to prevent HIV acquisition among this high-risk population.

\section{Trial status/registration}

At the time of submission, trial implementation had not yet begun. The trial is registered at ClinicalTrials.gov NCT03875950. All items from the WHO Trial Registration Data Set are available for this study on ClinicalTrials. gov.

\section{Author affiliations \\ ${ }^{1}$ Department of Global Health, University of Washington, Seattle, Washington, USA ${ }^{2}$ Department of Epidemiology, University of Washington, Seattle, Washington, USA \\ ${ }^{3}$ Research and Programs, Kenyatta National Hospital/University of Nairobi, Nairobi, Kenya \\ ${ }^{4}$ Department of Medicine, University of Washington, Seattle, Washington, USA ${ }^{5}$ Department of Pediatrics, University of Washington, Seattle, WA, United States ${ }^{6}$ Department of Biostatistics, University of Washington, Seattle, WA, United States ${ }^{7}$ Health Sciences Simulation \& Clinical Skills Center, Howard University, Seattle, Washington, DC, USA \\ ${ }^{8}$ Department of Child, Family, and Population Health Nursing, University of Washington, Seattle, Washington, USA}

Contributors PK and GJ-S are the principal investigators and they supervised the trial protocol development and implementation. JK is the site investigator and he oversaw Kenya-based implementation. PK, GJ-S, JK, KSW, BAR and JP participated in designing the study. TO helped to develop intervention materials and trained the standardised patient actors. BAR, KSW and PK were responsible for the statistical design of the trial. FA and HL oversaw field implementation of the trial. AL and KSW wrote the initial draft of the paper. All authors critically revised, read and approved the final manuscript.

Funding This work was supported by the National Institutes of Health grant number R01 HD094630.

Competing interests None declared.

Patient and public involvement Patients and/or the public were not involved in the design, or conduct, or reporting, or dissemination plans of this research.

Patient consent for publication Not required.

Provenance and peer review Not commissioned; externally peer reviewed.

Open access This is an open access article distributed in accordance with the Creative Commons Attribution Non Commercial (CC BY-NC 4.0) license, which permits others to distribute, remix, adapt, build upon this work non-commercially, and license their derivative works on different terms, provided the original work is properly cited, appropriate credit is given, any changes made indicated, and the use is non-commercial. See: http://creativecommons.org/licenses/by-nc/4.0/.

ORCID iD

Anna Larsen http://orcid.org/0000-0002-0096-9386

\section{REFERENCES}

1 Shannon K, Strathdee SA, Goldenberg SM, et al. Global epidemiology of HIV among female sex workers: influence of structural determinants. The Lancet 2015;385:55-71.

2 UNAIDS. UNAIDS data 20192019.

3 World Health Organization (WHO). Guidance on pre-exposure oral prophylaxis (PreP) for serodiscordant couples, men and transgender women who have sex with men at high risk of HIV: recommendations for use in the context of demonstration projects 2012. 
4 Thomson KA, Baeten JM, Mugo NR, et al. Tenofovir-based oral preexposure prophylaxis prevents HIV infection among women. Curr Opin HIV AIDS 2016;11:18-26.

5 Grant RM, Lama JR, Anderson PL, et al. Preexposure chemoprophylaxis for HIV prevention in men who have sex with men. N Engl J Med 2010;363:2587-99.

6 Choopanya K, Martin M, Suntharasamai P, et al. Antiretroviral prophylaxis for HIV infection in injecting drug users in Bangkok, Thailand (the Bangkok tenofovir study): a randomised, double-blind, placebo-controlled phase 3 trial. The Lancet 2013;381:2083-90.

7 Baeten JM, Donnell D, Ndase P, et al. Antiretroviral prophylaxis for HIV prevention in heterosexual men and women. N Engl J Med 2012;367:399-410.

8 Van Damme L, Corneli A, Ahmed K, et al. Preexposure prophylaxis for HIV infection among African women. N Engl J Med 2012;367:411-22.

9 Cowan FM, Delany-Moretlwe S, Sanders EJ, et al. Prep implementation research in Africa: what is new? J Int AIDS Soc 2016.

10 Dunbar MS, Kripke K, Haberer J, et al. Understanding and measuring uptake and coverage of oral pre-exposure prophylaxis delivery among adolescent girls and young women in sub-Saharan Africa. Sex Health 2018;15:513-21.

11 Celum CL, Delany-Moretlwe S, Baeten JM, et al. HIV pre-exposure prophylaxis for adolescent girls and young women in Africa: from efficacy trials to delivery. J Int AIDS Soc 2019;22.

12 Bender SS, Fulbright YK. Content analysis: a review of perceived barriers to sexual and reproductive health services by young people. Eur J Contracept Reprod Health Care 2013;18:159-67.

13 Godia PM, Olenja JM, Hofman JJ, et al. Young people's perception of sexual and reproductive health services in Kenya. BMC Health Serv Res 2014;14:172.

14 Godia PM, Olenja JM, Lavussa JA, et al. Sexual reproductive health service provision to young people in Kenya; health service providers experiences. BMC Health Serv Res 2013;13:476.

15 Hagey JM, Akama E, Ayieko J, et al. Barriers and facilitators adolescent females living with HIV face in accessing contraceptive services: a qualitative assessment of providers' perceptions in western Kenya. J Int AIDS Soc 2015;18:20123.

16 Godia PM, Olenja JM, Hofman JJ, et al. Young people's perception of sexual and reproductive health services in Kenya. BMC Health Serv Res 2014;14:1-13.

17 Kidia KK, Mupambireyi Z, Cluver L, et al. Hiv status disclosure to perinatally-infected adolescents in Zimbabwe: a qualitative study of adolescent and healthcare worker perspectives. PLoS One 2014;9:e87322.

18 Wachira J, Naanyu V, Genberg B, et al. Health facility barriers to HIV linkage and retention in Western Kenya. BMC Health Serv Res 2014;14:646.

19 Beima-Sofie K, Wagner AD, Pintye J, et al. Implementation challenges and strategies in integration of PreP into maternal and child health and family planning services: experiences of frontline healthcare workers in Kenya. International AIDS Society conference 2019.

20 Pilgrim N, Jani N, Mathur S, et al. Provider perspectives on PreP for adolescent girls and young women in Tanzania: the role of provider biases and quality of care. PLoS One 2018;13:e0196280.

21 Pimenta Oliveria MC, Bermudez XP, Godoi AM, et al. Qualitative evaluation of PrEP implementation in Brazil - ImPREP stakeholders study. International AIDS Society Conference 20192019.

22 Makoul G. Essential elements of communication in medical encounters: the Kalamazoo consensus statement. Acad Med 2001;76:390-3.

23 Kohler PK, Marumo E, Jed SL, et al. A national evaluation using standardised patient actors to assess STI services in public sector clinical sentinel surveillance facilities in South Africa. Sex Transm Infect 2017;93:247-52.

24 Wilson KS, Mugo C, Bukusi D, et al. Simulated patient encounters to improve adolescent retention in HIV care in Kenya: study protocol of a stepped-wedge randomized controlled trial. Trials 2017;18:619.

25 Daniels B, Dolinger A, Bedoya G, et al. Use of standardised patients to assess quality of healthcare in Nairobi, Kenya: a pilot, crosssectional study with international comparisons. BMJ Glob Health 2017;2:2.
26 Wafula F, Dolinger A, Daniels B, et al. Examining the quality of medicines at Kenyan healthcare facilities: a validation of an alternative post-market surveillance model that uses standardized patients. Drugs Real World Outcomes 2017;4:53-63.

27 Wilbur K, Elmubark A, Shabana S. Systematic review of standardized patient use in continuing medical education. $J$ Contin Educ Health Prof 2018;38:3-10.

28 Walker DM, Cohen SR, Fritz J, et al. Impact evaluation of PRONTO Mexico: a simulation-based program in obstetric and neonatal emergencies and team training. Simul Healthc 2016;11:1-9.

29 Walker DM, Holme F, Zelek ST, et al. A process evaluation of PRONTO simulation training for obstetric and neonatal emergency response teams in Guatemala. BMC Med Educ 2015;15:117.

30 Das J, Kwan A, Daniels B, et al. Use of standardised patients to assess quality of tuberculosis care: a pilot, cross-sectional study. Lancet Infect Dis 2015;15:1305-13.

31 Lyn Paget PH, Nedza S, Kurtz P, et al. Patient-Clinician communication: basic principles and expectations. NAM Perspectives 2019

$32 \mathrm{Mo} \mathrm{H}$. Guidelines on the use of antiretroviral drugs for treating and preventing HIV in Kenya 2016.

33 Mugwanya KK, Pintye J, Kinuthia J, et al. Integrating preexposure prophylaxis delivery in routine family planning clinics: a feasibility programmatic evaluation in Kenya. PLoS Med 2019;16:e1002885.

34 Nyblade L, Jain A, Benkirane M, et al. A brief, standardized tool for measuring HIV-related stigma among health facility staff: results of field testing in China, Dominica, Egypt, Kenya, Puerto Rico and St. Christopher \& Nevis. J Int AIDS Soc 2013:16.

35 Cox CL, McLaughlin RA, Steen BD, et al. Predicting and modifying substance use in childhood cancer survivors: application of a conceptual model. Oncol Nurs Forum 2006;33:51-60.

36 Rider EA, Hinrichs MM, Lown BA. A model for communication skills assessment across the undergraduate curriculum. Med Teach 2006;28:e127-34.

37 Ong LM, de Haes JC, Hoos AM, et al. Doctor-Patient communication: a review of the literature. Soc Sci Med 1995;40:903-18.

38 Kahn JA, Emans SJ, Goodman E. Measurement of young women's attitudes about communication with providers regarding Papanicolaou smears. J Adolesc Health 2001;29:344-51.

39 Stewart M. The Patient Perception of Patient Centeredness Questionaire (PPPC) \#04-1 - Centre for Studies in Family Medicine Western University 2019.

40 Woods ER, Klein JD, Wingood GM, et al. Development of a new adolescent patient-provider interaction scale (APPIS) for youth at risk for STDs/HIV. J Adolesc Health 2006;38:753.e1-7.

41 Dang B. Retaining HIV patients in care: the role of initial patient care experiences | SpringerLink 2019.

42 van Zanten M, Boulet JR, McKinley D. Using standardized patients to assess the interpersonal skills of physicians: six years' experience with a high-stakes certification examination. Health Commun 2007;22:195-205.

43 Programme NASC. Guidelines on antiretroviral drugs for treating preventing HIV infections in Kenya 2016.

44 Denno DM, Hoopes AJ, Chandra-Mouli V. Effective strategies to provide adolescent sexual and reproductive health services and to increase demand and community support. $J$ Adolesc Health 2015;56:S22-41.

45 MacPherson P, Munthali C, Ferguson J, et al. Service delivery interventions to improve adolescents' linkage, retention and adherence to antiretroviral therapy and HIV care. Trop Med Int Health 2015;20:1015-32.

46 UNAIDS. Women and HIV - a spotlight on adolescent girls and young women 2019.

47 Health RoKMo. National guidelines for provision of adolescent and youth friendly services in Kenya 2016.

48 Wolf HT, Halpern-Felsher BL, Bukusi EA, et al. "It is all about the fear of being discriminated [against]...the person suffering from HIV will not be accepted": a qualitative study exploring the reasons for loss to follow-up among HIV-positive youth in Kisumu, Kenya. BMC Public Health 2014;14:1154. 